\title{
Two-dimensional extreme skin depth engineering for CMOS photonics
}

\author{
Matthew van Niekerk ${ }_{9}^{1, *}$ Saman Jahani,${ }^{2,3}$ (1) Justin Bickford, ${ }^{4}$ Pak Cho, ${ }^{4}$ \\ Stephen Anderson,,${ }^{4,5}$ Gerald Leake, ${ }^{6}$ Daniel Coleman, ${ }^{6}$ Michael L. Fanto,,${ }^{1,7}$ \\ Christopher C. Tison, ${ }^{7}$ Gregory A. Howland, ${ }^{1,8}$ Zubin Jacob $_{9}{ }^{2}$ and Stefan F. Preble ${ }^{1}$ \\ ${ }^{1}$ Microsystems Engineering, Rochester Institute of Technology, Rochester, New York 14623, USA \\ ${ }^{2}$ School of Electrical and Computer Engineering and Birck Nanotechnology Center, Purdue University, West Lafayette, Indiana 47907, USA \\ ${ }^{3}$ Department of Electrical Engineering, California Institute of Technology, Pasadena, California 91125, USA \\ ${ }^{4}$ U.S. Army Research Laboratory, Adelphi, Maryland 20783, USA \\ ${ }^{5}$ Electrical, Computer and Systems Engineering Department, Rensselaer Polytechnic Institute, Troy, New York 12180, USA \\ ${ }^{6}$ State University of New York Polytechnic Institute, Albany, New York 12203, USA \\ ${ }^{7}$ Air Force Research Laboratory, Rome, New York 13441, USA \\ ${ }^{8}$ School of Physics and Astronomy, Rochester Institute of Technology, Rochester, New York 14623, USA \\ ${ }^{*}$ Corresponding author: mv7146@rit.edu
}

Received 7 December 2020; revised 24 February 2021; accepted 27 February 2021; posted 2 March 2021 (Doc. ID 416848 ); published 24 March 2021

\begin{abstract}
Extreme skin depth engineering (e-skid) can be applied to integrated photonics to manipulate the evanescent field of a waveguide. Here we demonstrate that e-skid can be implemented in two directions in order to deterministically engineer the evanescent wave allowing for dense integration with enhanced functionalities. In particular, by increasing the skin depth, we enable the creation of two-dimensional (2D) e-skid directional couplers with large gaps and operational bandwidth. Here we experimentally validate 2D e-skid for integrated photonics in a complementary metal-oxide semiconductor (CMOS) photonics foundry and demonstrate strong coupling with a gap of $1.44 \mu \mathrm{m}$. ( 2021 Optical Society of America under the terms of the OSA Open Access Publishing Agreement
\end{abstract}

https://doi.org/10.1364/JOSAB.416848

\section{EVANESCENT WAVES IN SILICON PHOTONICS}

Evanescent waves in silicon photonic waveguides have the propensity to cause parasitic optical cross talk. In traditional photonic circuits, design strategies must consider minimum separation distances between any closely spaced waveguides to prevent unwanted coupling [1]. This problem is inhibiting for many photonic circuits due to cost and size constraints. Many efforts have been made to overcome these issues, battling size constraints by employing inverse design [2,3] or implementing metamaterials to increase performance [4-6].

Recent work introduced a new, metamaterial paradigm for waveguiding that fundamentally suppresses coupling between waveguides [7]. In this approach, a subwavelength, multi-layer cladding is placed in plane and in parallel with the waveguide, decreasing the skin depth of the fundamental transverse-electric (TE) mode's evanescent field. The concept is called extreme skin depth engineering, or e-skid. E-skid has been employed as cross talk suppression $[7,8]$, and for high performance polarization splitting $[9,10]$. The e-skid features are created in the same processing step as the waveguide itself, allowing this to be an innate no-cost addition to any design. The addition of these features can reduce the cross talk between waveguides by more than 3 orders of magnitude, which will dramatically reduce the photonic design footprint [7].

Here we expand on this work by using e-skid engineering in two directions, within the same plane as the waveguide. Using both a parallel (as in [7]) and perpendicular e-skid cladding, we engineer the coupling between waveguides throughout a photonic circuit. Specifically, the perpendicular e-skid cladding (with features orthogonal to the waveguide) can dramatically increase the evanescent tail of the mode [or equivalently decrease its decay constant-as shown in Fig. 1(c)]. This then enables arbitrary enhancement in coupling between nearby waveguides anywhere in the circuit. While parallel e-skid cladding can be used to suppress coupling in the routing of the rest of the circuit. Here we explore this with the design of a two-dimensional (2D) e-skid directional coupler with a large gap $(\geq 1.4 \mu \mathrm{m})$ between the waveguides and large operational bandwidth $(\geq 40 \mathrm{~nm})$. We also demonstrate these 2D e-skid directional couplers in a complementary metal-oxide semiconductor (CMOS) photonic platform, thereby affirming the manufacturability of $e$-skid components and integration with foundry offerings. Ultimately, this work is motivated by the idea that we can employ $2 \mathrm{D}$ e-skid techniques to ensure low cross talk in 


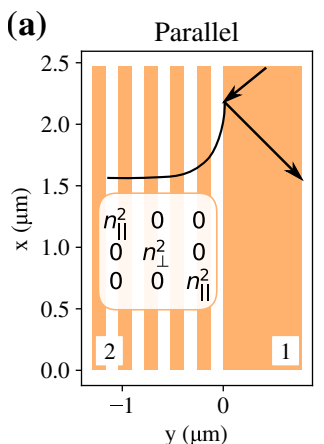

(b)

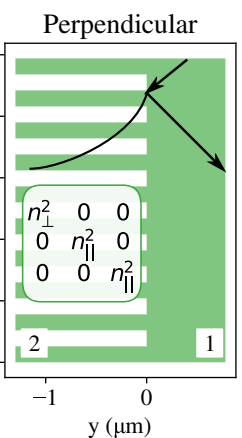

(c)

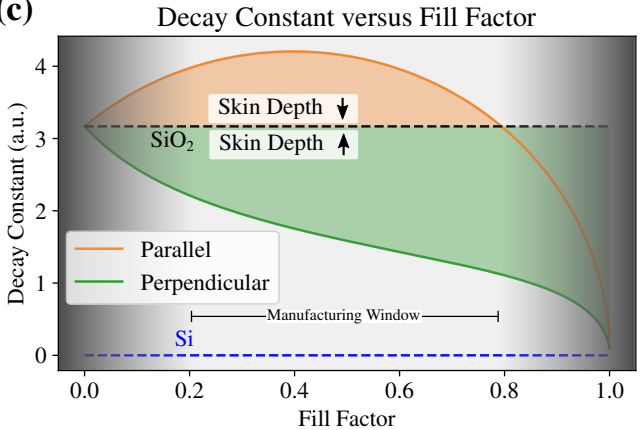

Fig. 1. (a) Here we demonstrate the parallel oriented subwavelength, multi-layer cladding. The anisotropic permittivity tensor is displayed over the cladding, which follows the Rytov relations for each direction. (b) The perpendicular cladding essentially swaps the $x x$ and $y y$ components of the permittivity tensor in panel (a). The incident wave is reflected (shown by the two arrows in medium one), and the evanescent wave is strongly decaying in medium two in panel (a) and weakly decaying in panel (b). (c) A plot of Eq. (3) for the two different cladding strategies. We see that decay is increased over $\mathrm{SiO}_{2}$ for most of the fill factors of the parallel case, whereas we can see a variable decrease in decay by almost the whole scale between the two materials in the perpendicular cladding. The likely "manufacturing window" over which these features can be fabricated in a CMOS silicon photonics foundry is indicated.

the routing phase (with parallel $e$-skid) and, without the use of bends, efficient coupling between waveguides with larger-thannormal gaps (with perpendicular $e$-skid) —fully leveraging both directions of e-skid. Consequently, 2D e-skid allows for dense integration within the constraints of CMOS manufacturing.

\section{THEORY}

\section{A. E-Skid in Two Directions}

Consider two media with index of refraction $n_{1}, n_{2}$. When an incoming wave from $n_{1}$ meets the boundary at $n_{2}$ and the angle is greater than the critical angle, $\theta_{i}<\theta_{c}=\sin ^{-1}\left(n_{2} / n_{1}\right)$, an evanescent wave is formed in the second medium. This wave does not carry power across the boundary; it exponentially decays into the second medium [11]. E-skid allows us to tune the decay constant of this evanescent wave by introducing subwavelength, periodic structures that transform a wave's (specifically, a polarized wave's) momentum $[12,13]$. These features change the second medium from an isotropic material to an anisotropic metamaterial. The anisotropy here refers to the permittivity values of the dielectric tensor of the material (where we are assuming that the permittivity can be defined $\epsilon_{r}=n^{2}$ ). For deep-subwavelength features, these component values are defined by the Rytov relations [14,15]:

$$
\begin{gathered}
n_{\|}^{2}=n_{1}^{2} \rho+n_{2}^{2}(1-\rho), \\
n_{\perp}^{-2}=n_{1}^{-2} \rho+n_{2}^{-2}(1-\rho),
\end{gathered}
$$

where $n_{1}, n_{2}$ are the indices of the first and second medium, respectively, and $\rho$ is the fill factor. The parallel component, $n_{\|}$, is defined in the direction parallel to the periodic structure's orientation, and the perpendicular component, $n_{\perp}$, is oriented perpendicular to the periodic structure. For features that are deep-subwavelength, these relations demonstrate how the second material transforms from isotropic to an anisotropic metamaterial; however, any subwavelength structures will exhibit anisotropy albeit without these neat relations.
The key result of the e-skid derivation leverages this anisotropy for the evanescent wave, which is characterized by the decay constant, $\beta$ :

$$
\beta\left(\theta_{i} ; \rho\right)=\frac{1}{\delta\left(\theta_{i}\right)}=k_{0} \frac{n_{2 x}(\rho)}{n_{2 y}(\rho)} \sqrt{n_{1}^{2} \sin ^{2}\left(\theta_{i}\right)-n_{2 y}(\rho)^{2}},
$$

where $k_{0}$ is the wavevector and $\theta_{i}$ is the angle of the incident wave to the boundary (we assume paraxial $\theta_{i} \approx \pi / 2$ ) [12]. The decay constant is now subject to a degree of variable tunability $(\rho)$, allowing for control of the evanescent wave $[7,12]$.

In Fig. 1(a), we show the dielectric tensor for the e-skid structure, where the periodicity of the subwavelength features is parallel to the boundary $(y=0)$. In this orientation, the diagonal components of the second material become $\left[n_{2 x}^{2}, n_{2 y}^{2}, n_{2 z}^{2}\right]=\left[n_{\|}^{2}, n_{\perp}^{2}, n_{\|}^{2}\right]$ in accordance with the Rytov relations [Eqs. (1) and (2)]. This structure will increase the decay constant of the evanescent wave, thereby decreasing the skin depth [7]. Without loss of generality, we recognize that we can rotate the optical axis by rotating the subwavelength features and realize $e$-skid in a second direction. Due to the direction dependency outlined by the Rytov relations, when we rotate the periodicity of the features, we effectively swap the $x x$ and $y y$ components of the dielectric tensor of the parallel cladding such that we now see $\left[n_{2 x}^{2}, n_{2 y}^{2}, n_{2 z}^{2}\right]=\left[n_{\perp}^{2}, n_{\|}^{2}, n_{\|}^{2}\right]$ [Fig. 1(b)]. The values of $n_{2 x}, n_{2 y}$ in Eq. (3) control the decay constant, and by rotating the periodic structure, we are able to dictate an increase or decrease.

We populated Eq. (3) with the new dielectric tensor values outlined in Figs. 1(a) and 1(b) such that we show in Fig. 1(c) the full range of decay constant tunability of e-skid in two directions. Figure 1(c) shows clearly that both decreasing and increasing skin depth can be achieved by the parallel features; however, applying this to CMOS photonics manufacturing, we generally omit the higher and lower fill factors due to resolution constraints [16]. We used a material platform consistent with CMOS photonics in Fig. 1(c), such that material one is silicon $(\mathrm{Si})$ and material two is silicon dioxide $\left(\mathrm{SiO}_{2}\right)$; however, this is true for any optical material combination as long as $n_{1}>n_{2}$. 


\section{B. E-Skid in Two Directions in Waveguides}

Optical waveguiding is not fully described by the simple electromagnetic wave-at-a-boundary example above. While it lends intuition, we must find the electromagnetic mode of the entire structure to get a clear picture of this effect. We used a commercial finite difference eigenmode (FDE) solver to simulate the TE modes of three specific types of waveguides to demonstrate e-skid in two directions [17]. Figure 2(a) shows a top view of a single-mode strip waveguide, where the propagation is in the $\hat{x}$ direction. Next to the strip waveguide, Fig. $2(\mathrm{~d})$ shows a $2 \mathrm{D}$ mode profile of the fundamental TE propagating mode. We introduce the wave supressing $e$-skid features on one side of the waveguide in Fig. 2(b) and show the corresponding 2D mode profile in Fig. 2(e). Finally, we introduce the wave enhancing e-skid features in Fig. 2(c) and the corresponding 2D mode profile in Fig. 2(f). We compiled the cross sections of all three modes in Fig. $2(\mathrm{~g})$ to demonstrate the effect of the features on the evanescent wave of the mode. Figure $2(\mathrm{~g})$ clearly demonstrates, with a log-scale in $y$, that the decaying wave outside of the center of the waveguide is suppressed by the parallel features, and greatly enhanced by the perpendicular features.

We extend our analysis to include a full wave threedimensional (3D) finite-difference time-domain (FDTD) solver with Bloch-periodic boundary conditions [17]. Figure 3 displays the decay constant as extracted for varying fill factors and periods of subwavelength features. The decay constant extraction fit for both Figs. 3(a) and 3(b) had average correlation coefficients of $R^{2}=0.98$. In Fig. 3(a), we show the results of the parallel cladding, and in Fig. 3(b), we show the perpendicular, both of which follow the general shape outlined by the analytical expression in Eq. (3) at the paraxial limit, or simply, a guided wave. As the period increases, we notice a shift in behavior of the peak toward the lower fill factors; however, this is not significant enough to alter the design parameters. We also note the similarity of Figs. 3 and 1(c), where the parallel features are the only features that increase the decay constant and the perpendicular features allow for deterministic reduction of the decay constant, thereby enhancing cross talk. Figure 3 stops at $270 \mathrm{~nm}$ to allow each simulation to fall below the Bragg limit. We note that the operational window for subwavelength devices is dependent on the minimally reliable feature (or hole) size for the manufacturing process. Here, we see at the highest period that only $30-70 \%$ of fill factors are expected to resolve for an $80 \mathrm{~nm}$ minimum.
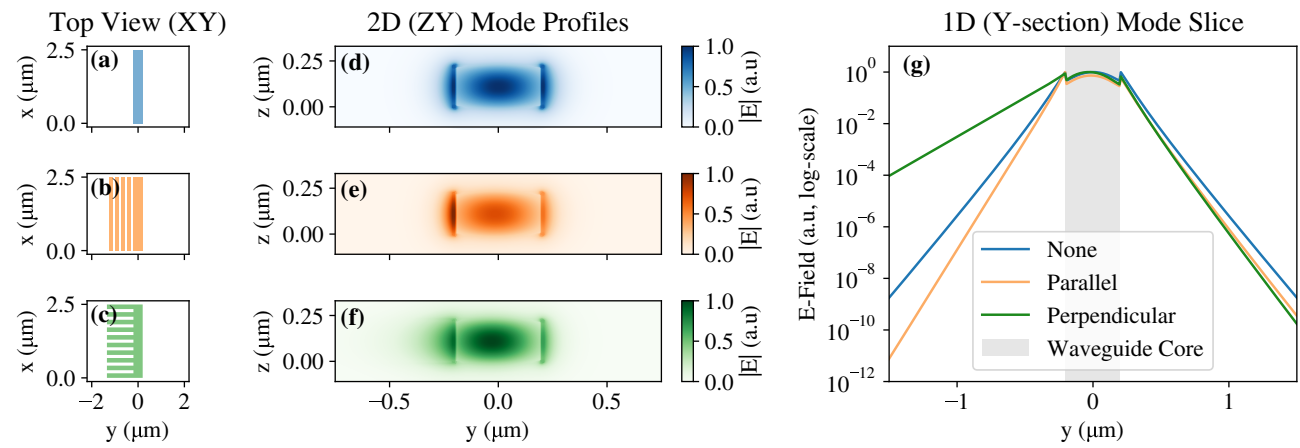

Fig. 2. Comparison of the different cladding strategies discussed, with the note that they have a common cladding on the right-hand side (normal isotropic $\mathrm{SiO}_{2}$ ). (a)-(c) Here we have the top-down perspective of the waveguides, which shows the cladding for the none, parallel, and perpendicular structures on the left-hand side, respectively. (d)-(f) Z-Y plane cross section mode profiles corresponding to the cladding diagrams from panels (a)-(c) where the width of the waveguides is $400 \mathrm{~nm}$ and the height is $220 \mathrm{~nm}$. (g) Center slice through each of the mode profiles, which demonstrate, on a log-linear scale, the amount of control we can impose on the evanescent wave with these structures. Simulations were done with an anisotropic material following Rytov relations [Eqs. (1) and (2)], where the core waveguide width was $400 \mathrm{~nm}$, and the fill factor was 0.6 for both orientations.
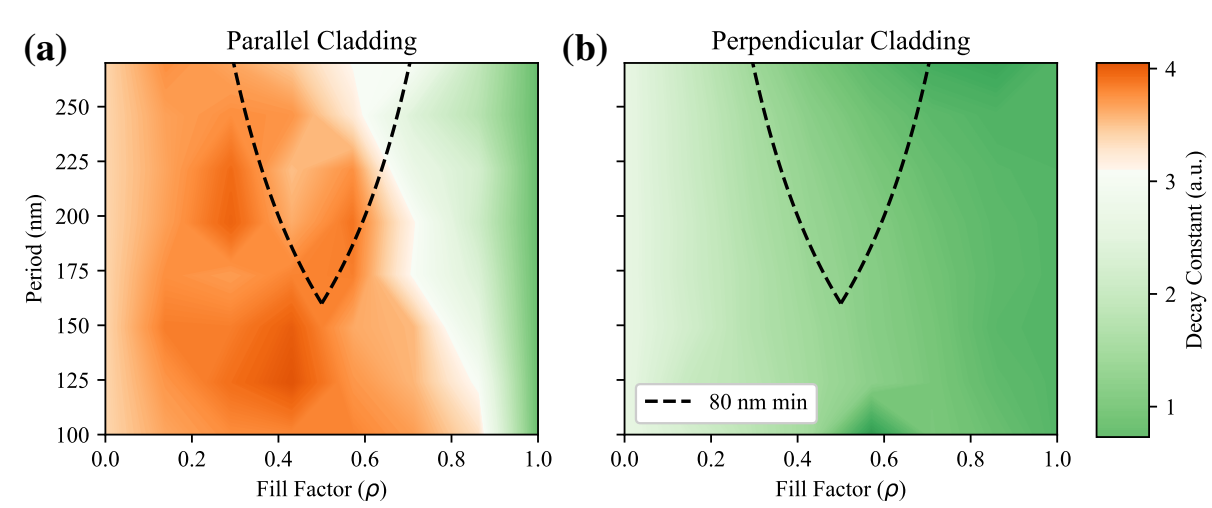

Fig. 3. Decay constant contour plots extracted via simulation for the (a) parallel and (b) perpendicular cladding features over varying fill factors and periods. We include a trace that indicates an $80 \mathrm{~nm}$ minimum feature/space cutoff to demonstrate the constraints present in subwavelength CMOS photonics. These simulations were run with waveguide widths of $400 \mathrm{~nm}$. 


\section{2D E-SKID DIRECTIONAL COUPLER DESIGN}

We propose and demonstrate a new coupler that leverages e-skid in two directions to create coupling in desired regions. In a traditional integrated photonic platform, a directional coupler is created by bending two waveguides close to each other [Fig. 4(a)]. The waveguides must otherwise be kept far apart in other parts of a circuit in order to avoid unwanted coupling, limiting the circuit density. By using $e$-skid with parallel subwavelength features [Fig. 4(b)] that suppress coupling, we overcome this limitation and keep two waveguides within close proximity, with negligible coupling. Furthermore, when the design with e-skid needs coupling, we show that the introduction of perpendicular subwavelength features in the coupling region, as are seen in Fig. 4(c), will significantly enhance coupling. These features have tunable variables (i) period $\left(\Lambda_{\perp}\right)$ and (ii) fill factor $\left(\rho_{\perp}\right)$, which directly tune the amount of coupling experienced. We introduce $2 \mathrm{D} e$-skid as a way to leverage the size reduction offered by the parallel features with the addition of the perpendicular features to create practical circuits as seen in Fig. $4(\mathrm{~d})$.

\section{A. Coupled Modes for E-Skid}

The evanescent wave of the mode, even though it carries no power across the boundary, causes coupling between parallel guides if the overlap between the evanescent waves of supported modes is large enough [18]. From coupled mode theory [18], we define the power in the bar and cross ports as

$$
\begin{aligned}
& P_{b a r}(L)=P_{0} \cos ^{2}(\kappa L)=P_{0} \cos ^{2}\left(\frac{\pi}{2} \frac{L}{L_{x}}\right), \\
& P_{\text {cross }}(L)=P_{0} \sin ^{2}(\kappa L)=P_{0} \sin ^{2}\left(\frac{\pi}{2} \frac{L}{L_{x}}\right),
\end{aligned}
$$

with $L$ as the coupling length, $P_{0}$ as the injected power, $\kappa$ as the coupling coefficient, and $L_{x}$ as the crossover length such that $\kappa=\pi /\left(2 L_{x}\right)$, and the bar and cross refer to the light remaining in the injected waveguide or transitioning to the other waveguide, respectively. The crossover length is defined so that when $L=L_{x}$, there is complete power transfer from waveguide one to two.

This approach allows for an intuitive understanding of the device. The crossover length is given as

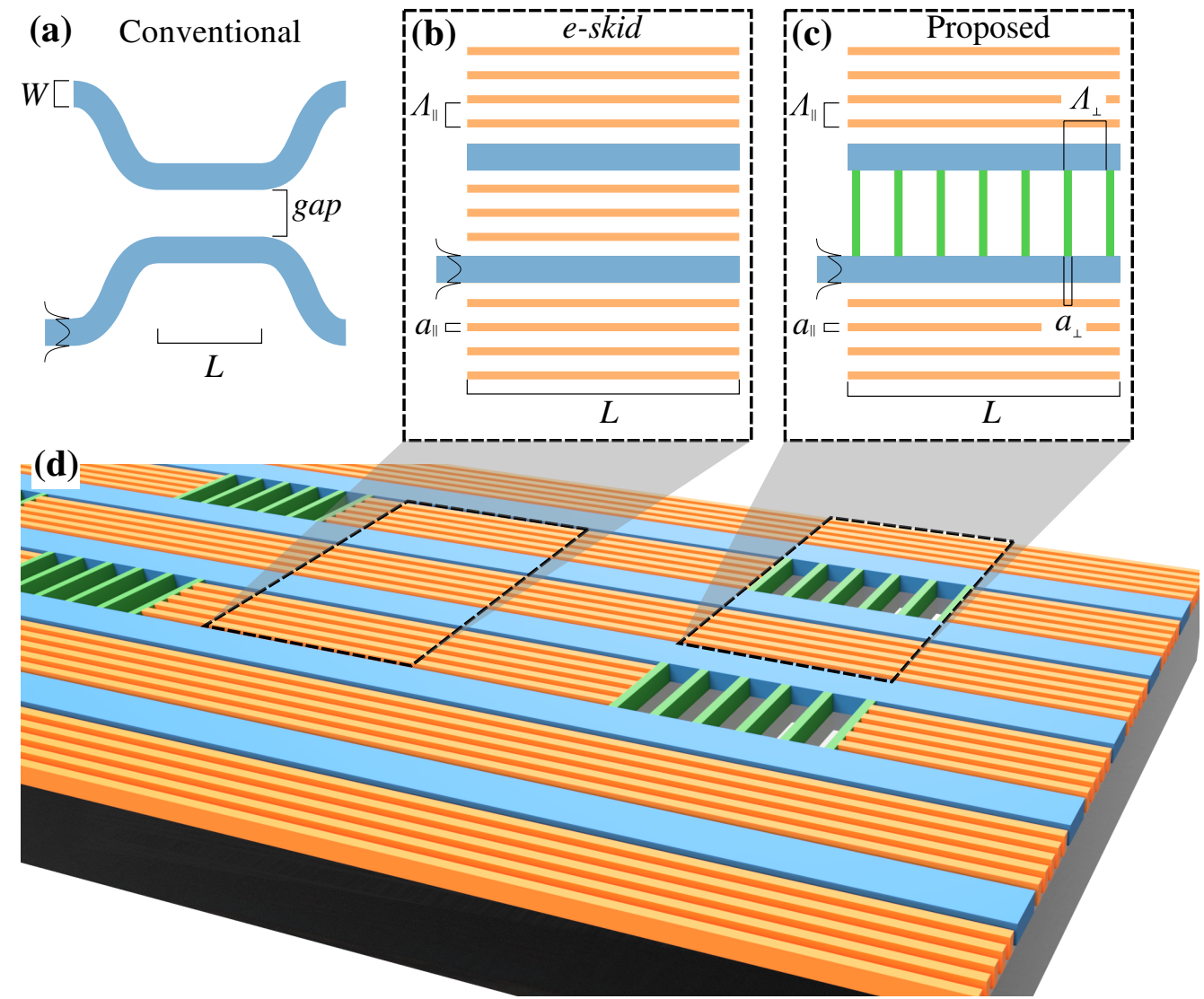

Fig. 4. (a) Conventional directional coupler with a coupling region characterized by the gap between waveguides and the length of the parallel section. (b) E-skid platform discussed in [7], where the period $\left(\Lambda_{\|}\right)$and silicon fill $\left(a_{\|}\right)$characterize the subwavelength features. (c) Our directional coupler that leverages the enhancing e-skid features in the coupling region, where the features outside the coupling region are the same as panel (b) and where the period $\left(\Lambda_{\perp}\right)$ and silicon fill $\left(a_{\perp}\right)$ characterize the subwavelength features in the coupling region. (d) Example of an integrated photonic circuit implementing 2D e-skid. Note the circuit maintains the size reduction of e-skid coupled with 2D e-skid directional couplers. In the colored version, the colors throughout indicate the photonic waveguides (blue), parallel (orange), and perpendicular (green) e-skid features (where all are the same material—namely, silicon), and the base is the buried oxide (black). The figure is not drawn to scale. 


$$
L_{x}=\frac{\lambda}{2\left(n_{\mathrm{even}, \lambda}-n_{\mathrm{odd}, \lambda}\right)},
$$

where $\lambda$ is the free space wavelength and $n_{\text {even }, \lambda}, n_{\text {odd }, \lambda}$ are the effective indices of the even and odd modes, respectively [1]. The field of the odd mode is antisymmetric across the coupling region, and it remains generally unaffected by symmetric features there [19]. However, by introducing features into the coupling region, the even mode is affected, thereby enabling dispersion engineering of the directional coupler-specifically, controlling the directional couplers' optical bandwidth [19]. By crafting $L_{x}$, we can dictate how the device performs according to Eqs. (4) and (5). Essentially, if we make the slope of $L_{x}$ as flat as possible over a span of $\lambda$, we ensure a useful operating bandwidth (e.g., a $3 \mathrm{~dB}$ coupler) is preserved for that span. Our design is fundamentally different than [19] due to the structural asymmetry, which encourages coupling, and the higher fill factor. These parameters allow us to create a directional coupler with more than an order of magnitude shorter crossover length in comparison, at the penalty of reduced operating bandwidth.

In order to demonstrate the effect of dispersion engineering, we begin by simulating the photonic band structure of the directional coupler in a full wave 3D FDTD solver with Blochperiodic boundary conditions [17]. Figures 5(a)-5(c) show the photonic band structure of a traditional, one-dimensional (1D) $e$-skid and 2D e-skid directional coupler. These directional couplers are fundamentally different from photonic crystals as they are not designed to work in the photonic bandgap; instead, these subwavelength features allow for low loss propagation through the periodic structures below the bandgap [15]. The traditional and e-skid couplers exhibit similar band structures, but the 2D e-skid directional coupler's even mode is approaching the band edge just above $200 \mathrm{THz}(1500 \mathrm{~nm})$. Because the even mode is near the band edge, dispersion is increased, which allows for flexibility in tuning the behavior.
From the band structures, we extract the dispersive effective index of both the fundamental even and odd supermodes [Figs. 5(d)-5(f)]. The effective indices exhibit a similar characteristic shape to their corresponding band structures. The e-skid coupler brings the even mode effective index much closer to the odd mode in comparison with the traditional coupler, and the $2 \mathrm{D}$ e-skid directional coupler has increased the difference between the two effective indices. Figures $5(\mathrm{~g})-5(\mathrm{i})$ show the crossover length given the corresponding effective indices. The traditional coupler and e-skid behave as expected, with an increase in crossover length for the latter. The $2 \mathrm{D}$ e-skid directional coupler exhibits a dramatically reduced crossover length and, in relation to dispersion engineering, a completely different shape. It is important to note that the $2 \mathrm{D}$ e-skid directional coupler does support a higher-order mode [Fig. 5(c)]; however, the coupling efficiency extracted from a modal overlap integral between the fundamental and the first higher-order mode is $<8 \%$ over the wavelength span for our designs, according to our 3D FDTD simulations [17]. While $8 \%$ is not insignificant, tweaking our parameters (specifically $\rho_{\perp}$ and $\Lambda_{\perp}$ ) can reduce this coupling efficiency into higher-order modes, increasing device performance [19]. For this experiment, the primary design choices were dictated from a perspective of manufacturability. In the future, small decreases to $\rho_{\perp}$ and $\Lambda_{\perp}$ will result in higher performing couplers verified by our $3 \mathrm{D}$ FDTD simulations [17] and prior work [19]. Additionally, careful consideration of tapering, which is not investigated in this work, can also mitigate the excitation of higher-order modes $[15,20]$.

The $2 \mathrm{D} e$-skid directional coupler is fundamentally different to a multimode interferometer (MMI). MMIs offer compact power splitting via a self-imaging effect that manifests from the excitation of higher-ordered modes, creating an interference pattern that repeats at a length specific to the physical parameters $[21,22]$. The $2 \mathrm{D}$ e-skid directional coupler specifically (a) Photonic Bandstructure

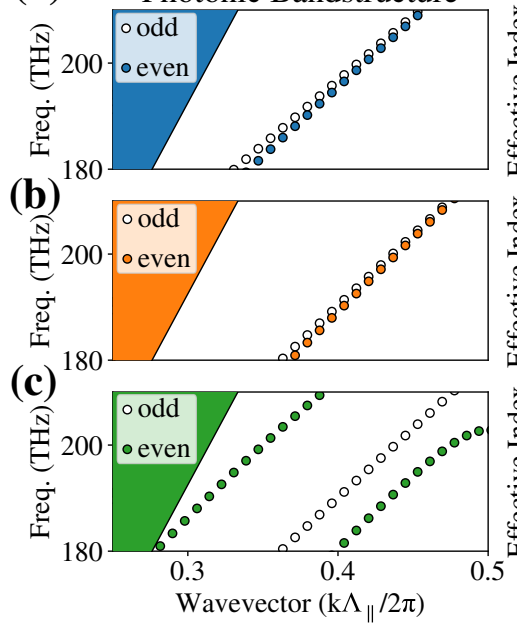

(d) Effective Indices
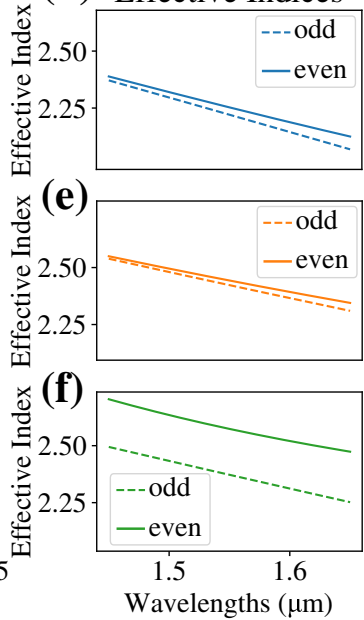
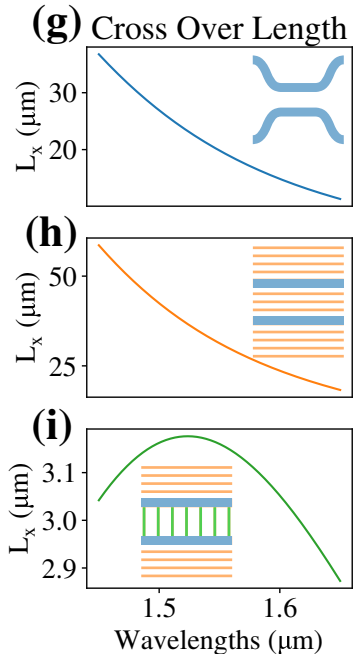

Fig. 5. (a)-(c) Photonic band structures of the conventional, 1D e-skid, and 2D e-skid directional couplers from Figs. 4(a)-4(c), respectively. (d)(f) Extracted effective indices of the band structures from panels (a)-(c), respectively. (g)-(i) Crossover length, calculated from Eq. (6). The insets of panels (g)-(i) show the device diagrams for each type of coupler. These devices were all simulated with the same gap to illustrate the effects on the same scale. In practice, the gap is limited by (i) the fabrication process, (ii) the circuit application, and (iii) the length of the waveguides. Therefore, the gap is often larger than shown here. The e-skid design parameters were gap $=270 \mathrm{~nm}, \Lambda_{\|}=50 \mathrm{~nm}, \rho_{\|}=50 \%, \Lambda_{\perp}=270 \mathrm{~nm}, \rho_{\perp}=50 \%$, and $W=$ $400 \mathrm{~nm}$. 
operates as a directional coupler, where the characteristics of the device arise from the coupled supermodes supported by the two waveguides [18]. The perpendicular, metamaterial features act to control the decay constant of the two modes' evanescent tails by shaping the material between the waveguides, enabling $2 \mathrm{D}$ e-skid and dispersion engineering, as demonstrated by the simulation results in Fig. 3.

\section{B. Device Design}

We investigate the effect of different parameter variations of the $2 \mathrm{D}$ e-skid directional coupler. Figure 6 shows the results of varying the fill factor, period, and gap between waveguides. These parameter variations indicate the substantial tunability offered by two-directional e-skid. First, we selected the operating gap between the two waveguides to be $1.44 \mu \mathrm{m}$ in order to stay consistent with the parallel e-skid features $\left(\rho_{\|}=60 \%, \Lambda_{\|}=225 \mathrm{~nm}\right.$, six layers deep results in a 1.44 $\mu \mathrm{m}$ gap). We designed these devices for manufacturing with the American Institute of Manufacturing (AIM) Photonics CMOS foundary Multi-Project Wafer (MPW) offering. For a photolithographic process like this one, we must take in to account the limitations of the processing, like feature size. For example, many prior work designs with features smaller than $60 \mathrm{~nm}$ would not resolve with CMOS processing compared to electron beam lithography. We chose to design our devices with $\Lambda_{\perp}=275 \mathrm{~nm}$ to remain beneath the Bragg limit but maintain high manufacturing quality. The parallel features were designed with $\Lambda_{\|}=225 \mathrm{~nm}$. It should be noted that the parallel cladding structures are less challenging for a lithographic system because they are lines, not holes [16]. We targeted $\rho_{\perp}=60 \%$ for the majority of our devices because we assumed that the features would be over etched, a common practice in siliconon-insulator fabrication, so that the fill factor would decrease [23].

\section{2D E-SKID DIRECTIONAL COUPLER DEVICE MEASUREMENTS AND PARAMETER EXTRACTION}

\section{A. Experiment}

Our experimental setup is shown in Fig. 7(a). We placed the chip on a mount in between two 3-axis stages with bare fiber on either side for coupling in and out. For the input, we connected the fiber to a tunable laser source (TLS) and a polarization controller (PC) to ensure TE polarization, and we measured the output signal with an optical powermeter (OPM). The fibers were edge coupled to the chip, which routed the light through strip/wire waveguides to the devices. To transition from the strip waveguide mode to the e-skid's, we slowly introduced the parallel and perpendicular claddings as seen in Fig. 7(c) and depicted in the schematic in Fig. 7(a). We were careful to design simple tapers because when periodic, asymmetric features are introduced, there is a chance for radiative losses [15]. We measured total device loss at $\leq 2 \mathrm{~dB}$ per device, which can be imrpoved with longer tapers or carefully designed width-varying tapers to ensure smooth modal transition.

We collected the transmission spectra [an example is shown in Fig. 7(b)]. The spectrum shows a characteristic "chirp"-like behavior, which is due to the dispersive nature of the crossover length. This is seen in Fig. 6, where, based on the perpendicular e-skid parameters of the device, the crossover length can exhibit a significant change with wavelength. This will result in a quickly oscillating output [given by Eqs. (4) and (5)]. With that said, using the experimental measurements in conjunction with the previously described models, we were able to extract the parametric dependence of the coupler designs.

\section{B. Parameter Extraction Method}

After extracting the transmission spectra from our fabricated devices, we used a dispersive model for extracting the crossover length from the data. Because inverse sine functions are multivalued, we cannot obtain $L_{x}$ directly from Eq. (4) or Eq. (5). We instead employed the behavioral model for characterizing directional couplers [24]. For the crossover length, we are interested
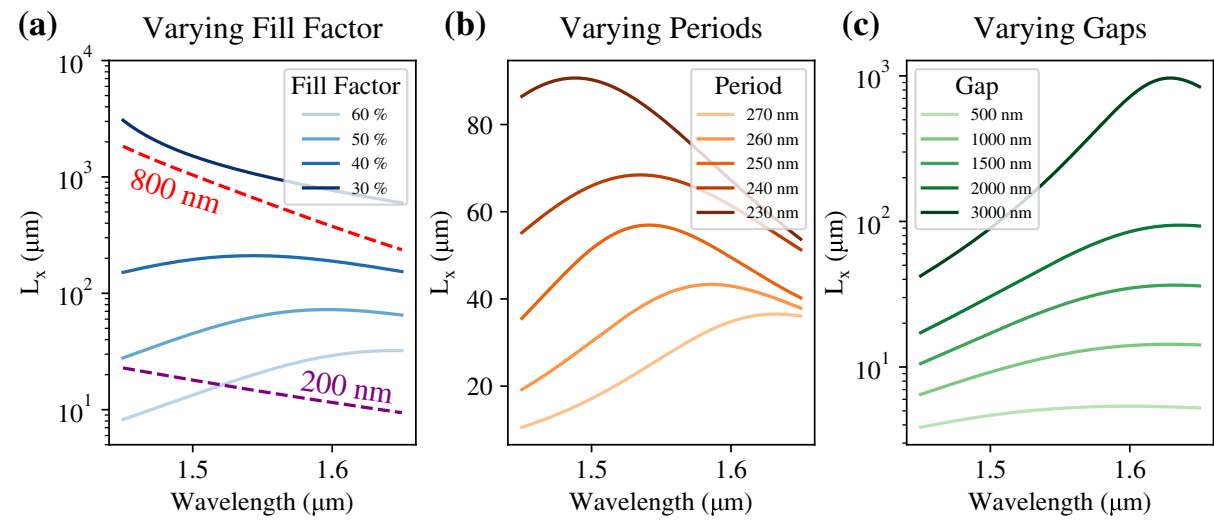

Fig. 6. Dispersive plots representing the crossover length for different varying parameters. (a) Fill factor sweep with period and gap fixed at $270 \mathrm{~nm}$ and $1500 \mathrm{~nm}$, respectively. The reference lines indicate the crossover length traditional directional coupler with gaps of $200 \mathrm{and} 800 \mathrm{~nm}$. (b) Period sweep with fill factor and gap fixed at $60 \%$ and $1500 \mathrm{~nm}$, respectively. (c) Coupling gap sweep with period and fill factor fixed at $270 \mathrm{~nm}$ and $60 \%$, respectively. 


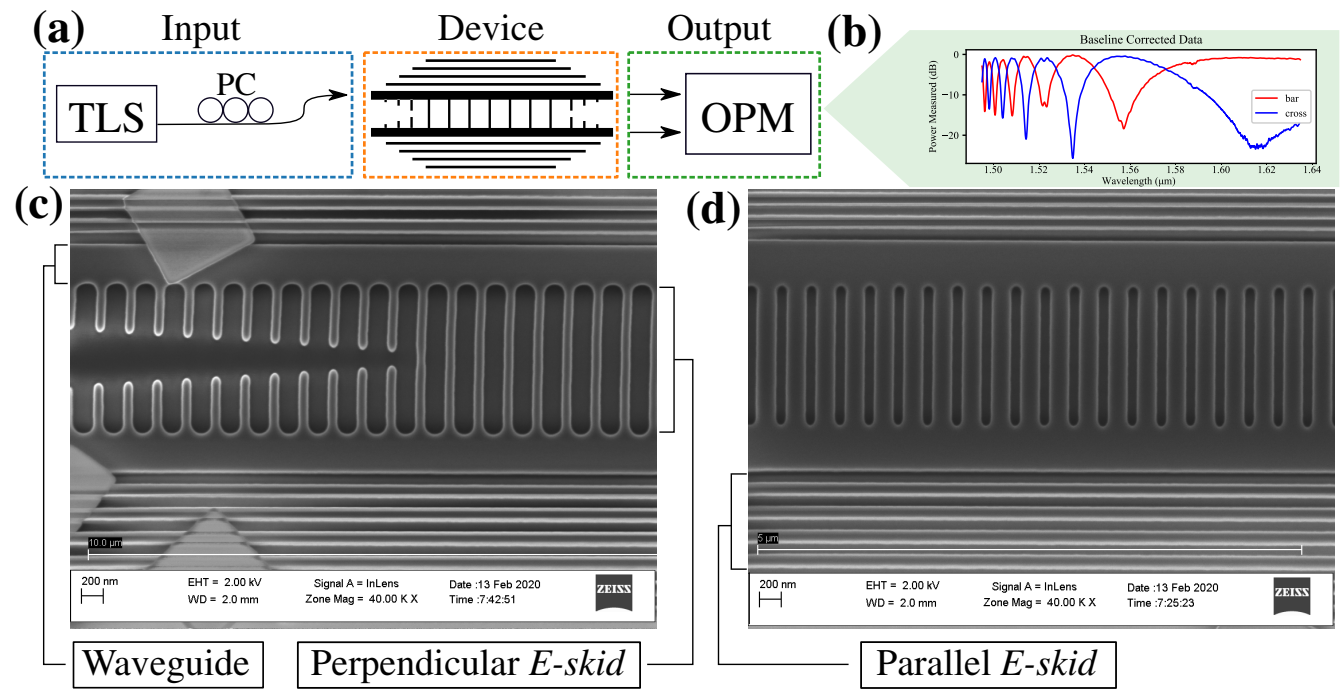

Fig. 7. (a) Experimental setup. A tunable laser source (TLS) is connected to the device via a polarization controller (PC). The outputs of the device are then connected to an optical powermeter (OPM). (b) An example spectrum from the measured data. (c) and (d) Scanning electron microscope (SEM) image of two different 2D e-skid directional couplers fabricated by AIM photonics, focused on the taper from the strip waveguides used to couple (c) to optical fibers and (d) in the center of the device. The objects are debris from the oxide-release etch for the SEM.

in the wavelength dependence, so we prepared the data by filtering the noise and normalizing the bar and cross measurements. We used a polynomial expansion of the coupling coefficient coupled with a non-linear least squares (NLS) optimization algorithm to find the best fit for $L_{x}[17,25,26]$. Because many of our devices exhibits a strong "chirp-like" behavior [Fig. $7(\mathrm{~b})$ ], we used a third-order polynomial expression for $L_{x}$ to determine the best fit, such that

$$
L_{x}(\lambda)=L_{x, 0}+L_{x, 1} \lambda+L_{x, 2} \lambda^{2}+L_{x, 3} \lambda^{3}
$$

where the curve is characterized by fitting parameters $L_{x, 0}, L_{x, 1}, L_{x, 2}, L_{x, 3}$ pertaining to the wavelength, $\lambda$. The NLS optimization aimed to minimize the difference between the measured and theoretical spectra by adjusting the fit parameters in Eq. 7 .

\section{Experimental Results}

In Fig. 8(a), we show the device's dependence on fill factor variation. Fill factors up to $60 \%$ were successfully fabricated in the CMOS process and will be reported below, while fabrication specific optimization needs to go into higher fill factor devices. The fill factor variations resemble those from the simulations [Fig. 6(a)], where higher fill factors increased $L_{x}$. In Fig. 8(b), we investigated coupler-length variation for a fixed fill factor $(60 \%)$ and gap $(1.44 \mu \mathrm{m})$. We fit nine different directional couplers with the exact same parameters changing only the coupling length. According to Eqs. (4) and (5), the length is independent of the coupling coefficient, $\kappa$, and therefore these couplers should exhibit identical $L_{x}$ measurements. There are manufacturing variations, measurement errors, and fitting errors that reveal themselves in Fig. 8(b). The inset shows a 95\% confidence interval for these nine couplers' $L_{x}$ extraction and showcases expected similar behavior for all of these couplers.
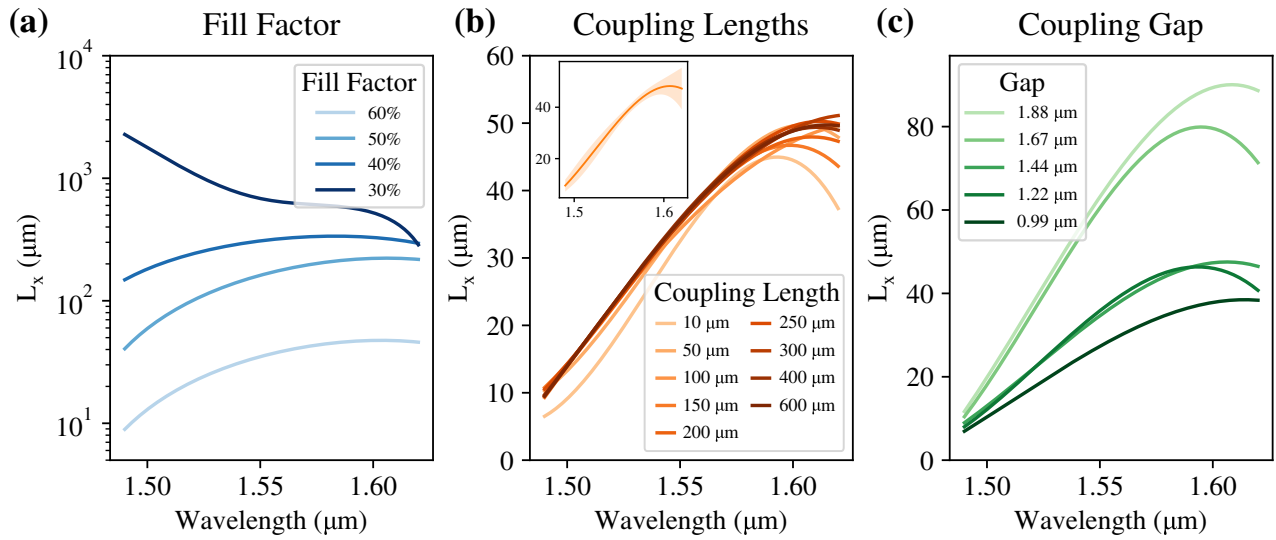

Fig. 8. (a) Experimental extraction of $L_{x}$ for the varying fill factors. (b) Experimental extraction of $L_{x}$ for the varying coupling lengths. Here we expect that the values will be similar. (c) Experimental extraction of $L_{x}$ for the varying coupling gaps, indicating an average change of $L_{x}$, but less conclusive over the range. 
Experimental and Theoretical Coupler Spectra

$\begin{array}{ll}\text { (a) Experimental 100/0 Coupler(b) Theoretical 50/50 } & \text { (c) Theoretical } 1.55 \mu \mathrm{m} 50 / 50\end{array}$

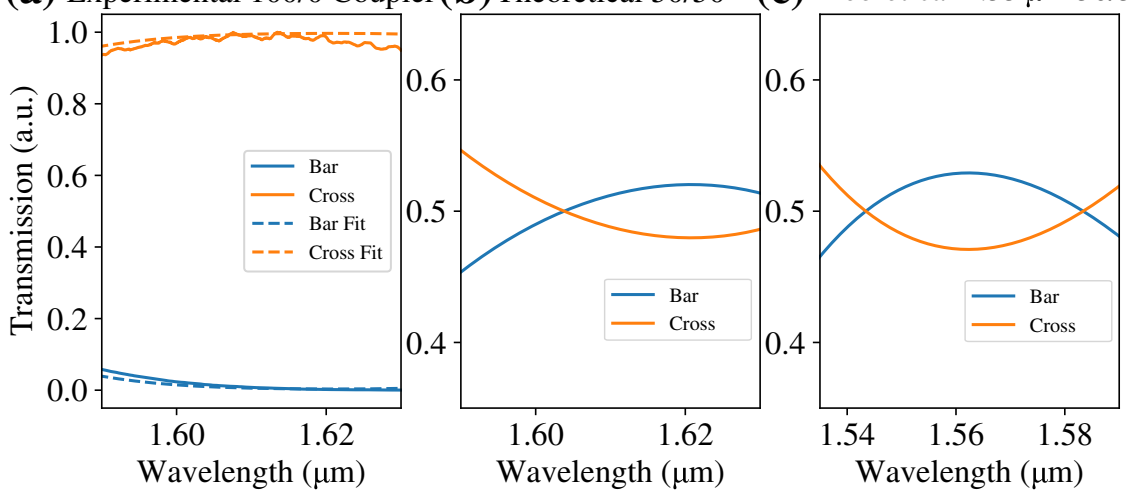

Fig. 9. (a) Experimental and fitting results for an $L_{x}$ coupler (100/0) splitting ratio. (b) Based on the experimental results in panel (a), we propose a $50 / 50$ splitter operating from 1.58 to $1.62 \mu \mathrm{m}$. (c) Based on simulation results from Fig. 6(a), we propose a 50/50 splitter operating from 1.54 to 1.58 $\mu \mathrm{m}$.

This truly highlights the utility of these devices, as they are able to couple fully in $\leq 50 \mu \mathrm{m}$, even with a large gap of $1.44 \mu \mathrm{m}$. Finally, Fig. 8(c) shows the $L_{x}$ extraction for varying gaps. Even though there is a qualitative match between the simulations and experimental results, manufacturing variations account for the quantitative differences.

\section{FUTURE WORK}

In this work, we performed a comprehensive study of the parameter space of our proposed directional coupler using 2D e-skid. In the future, it will be desirable to realize devices with particular performance characteristics. From our experimental data (Fig. 8 ), we extracted that we are able to realize a $2 \mathrm{D}$ e-skid directional coupler that achieves $100 \%$ coupling (100/0 splitting ratio) with a coupling length of $L=50 \mu \mathrm{m}$ as seen in Fig. 9(a), using $\rho_{\perp}=60 \%, \Lambda_{\perp}=275 \mathrm{~nm}$, and coupling gap of $1.44 \mu \mathrm{m}$. We can take this to design a 50/50 directional coupler. Figure 9(b) shows the theoretical transmission spectrum of this device. We set the coupling length $L=\operatorname{avg}\left(L_{x}\right) / 2=23.5 \mu \mathrm{m}$, and we see broadband behavior of nearly $40 \mathrm{~nm}$. Additionally, we can slightly vary parameters to tune the device to a more desirable center wavelength. For example, by reducing the period of the $2 \mathrm{D}$ e-skid directional coupler to $\Lambda_{\perp}=255 \mathrm{~nm}, \rho_{\perp}=50 \%$, and coupling gap of $1.44 \mu \mathrm{m}$, the device's operating band can now be centered closer to $1.55 \mu \mathrm{m}$ and achieves an even larger operating bandwidth of $>40 \mathrm{~nm}$ [Fig. 9(c)], which is sufficient for many applications.

\section{CONCLUSION}

We introduced the deterministic, targeted control of the evanescent wave in the TE mode of strip waveguides by employing $e-s k i d$ features in two directions. We designed and demonstrated 2D e-skid directional couplers on a CMOS photonic chip fabricated by AIM Photonics. All of the results were compared to simulations by extracting design parmaters, using a NLS optimization technique coupled with a behavioral model of the directional coupler [24]. With the parameter extraction, we show experimentally that $e$-skid waveguides, and the $2 \mathrm{D}$ e-skid directional coupler in particular, are possible to realize in a CMOS platform. Moving forward, we can design full 2D e-skid circuits that achieve high densities by both suppressing and enhancing coupling at will, while operating with large bandwidths.

\section{APPENDIX A: VERIFYING MODE CONVERSION EFFICIENCY OF 1D E-SKID FOR CMOS PHOTONICS}

We verify the conversion efficiency between strip waveguides and $e$-skid waveguides by comparing the loss coefficients at the resonances of a racetrack resonator $[27,28]$. We designed three strip waveguide racetrack resonators, Fig. 10(a), with varying gaps, and then the exact same racetrack resonators in which we add two e-skid features into the ring, Fig. 10(c). The intent of this experiment is to quantify the additional loss created by these features, and thereby measure the mode conversion efficiency between strip and $e$-skid waveguides [7]. A simple ring resonator [shown in Fig. 10(a)] can be paramaterized by two coefficients, $\tau$, the self-coupling coefficient that indicates how much light goes through the coupler, and $\alpha$, the loss coefficient that indicates how much light is lost into the ring. We extract $\alpha$ and $\tau$ according to the method described by [27], such that

$$
\begin{gathered}
\mathcal{F} \equiv \frac{\Delta \lambda_{\mathrm{FSR}}}{\Delta \lambda_{\mathrm{FWHM}}}, \\
\mathcal{E} \equiv \frac{T_{\mathrm{MAX}}}{T_{\mathrm{MIN}}}, \\
A=\frac{\cos (\pi / \mathcal{F})}{1+\sin (\pi / \mathcal{F})}, \\
B=1-\left(1-\frac{\cos (\pi / \mathcal{F})}{1+\cos (\pi / \mathcal{F})}\right) \frac{1}{\mathcal{E}}, \\
(\alpha, \tau)=\left[\frac{A}{B}\right]^{1 / 2} \pm\left[\frac{A}{B}-A\right]^{1 / 2} .
\end{gathered}
$$


(a)

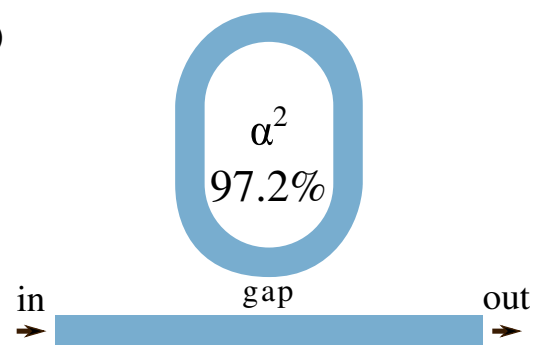

(c)

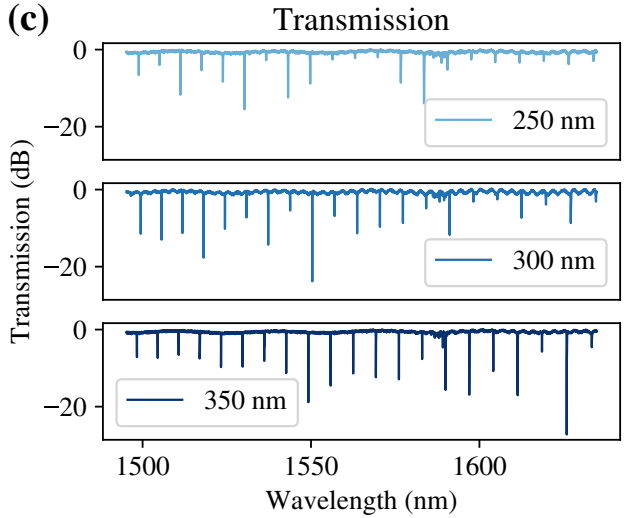

(b)

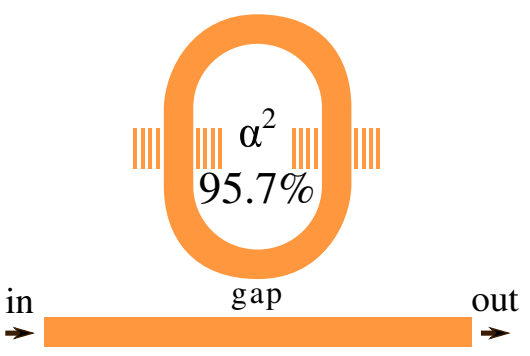

(d)

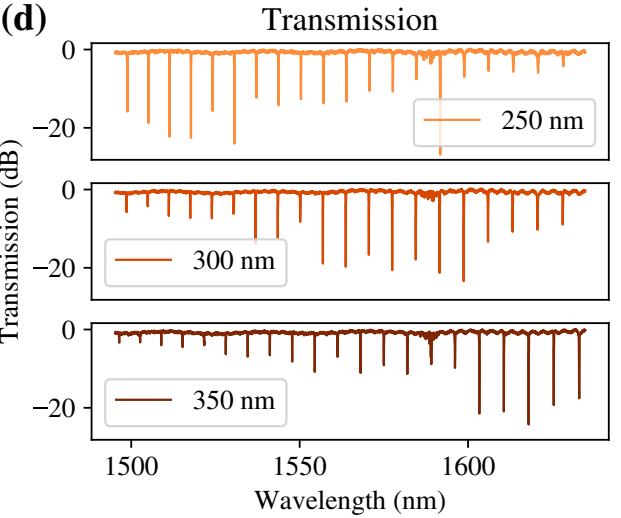

Fig. 10. (a) Strip waveguide racetrack ring resonator. We measured an average loss into the ring of $97.2 \%$ at the resonant peaks. (b) Transmission spectra for three equivalent strip waveguide racetrack ring resonators with varying coupling gaps of 250, 300, and $350 \mathrm{~nm}$. (c) Strip waveguide racetrack ring resonator with two parallel cladding features for comparison with panel (a). The loss into the ring was measured at $95.7 \%$ on average. (d) Transmission spectra for the three rings of the same varying gaps as panel (b) with the addition of the features.

The finesse, $\mathcal{F}$, is defined as the ratio between the free spectral range, $\Delta \lambda_{\mathrm{FSR}}$, and the full width at half-maximum, $\Delta \lambda_{\mathrm{FWHM}}$, of each resonance. The exctinction ratio, $\mathcal{E}$, is defined as the ratio between the transmission maximum, $T_{\mathrm{MAX}}$, off resonance, and the minimum, $T_{\mathrm{MIN}}$, at each resonance. We can decouple $\alpha, \tau$ in Eq. (A5) using the method further discussed in [27]. When we determine $\alpha$, we know that $\alpha^{2}$ indicates the percentage lost into the ring, which allows us to compare these two different resonators. This resulted in average values of $\alpha^{2}=97.2 \%$ and $\alpha^{2}=95.7 \%$ for the two ring types [Figs. 10(a) and 10(c), respectively]. This leads to a mode conversion efficiency of $99.6 \%$.

Funding. National Science Foundation (1810282); Air Force Research Laboratory (FA8650-15-2-5220, FA8750-16-2-0140).

Acknowledgment. Any opinions, findings, and conclusions or recommendations expressed in this material are those of the author(s) and do not necessarily reflect the views of the National Science Foundation. The views and conclusions contained herein are those of the authors and should not be interpreted as necessarily representing the official policies or endorsements, either expressed or implied, of Air Force Research Laboratory or the U.S. Government. M. v. N. and S. F. P. would like to acknowledge Navin B. Lingaraju for sparking a collaboration between RIT and Purdue.

Disclosures. The authors declare that there are no conflicts of interest related to this article.

\section{REFERENCES}

1. L. Chrostowski and M. Hochberg, Silicon Photonics Design: From Devices to Systems (Cambridge University, 2015).
2. A. Y. Piggott, J. Lu, K. G. Lagoudakis, J. Petykiewicz, T. M. Babinec, and J. Vučković, "Inverse design and demonstration of a compact and broadband on-chip wavelength demultiplexer," Nat. Photonics 9, 374-377 (2015).

3. B. Shen, P. Wang, R. Polson, and R. Menon, "An integratednanophotonics polarization beamsplitter with $2.4 \times 2.4 \mu \mathrm{m} 2$ footprint," Nat. Photonics 9, 378-382 (2015).

4. S. Jahani and Z. Jacob, "All-dielectric metamaterials," Nat. Nanotechnol. 11, 23-36 (2016).

5. J. M. Luque-González, A. Herrero-Bermello, A. Ortega-Moñux, Í. Molina-Fernández, A. V. Velasco, P. Cheben, J. H. Schmid, S. Wang, and R. Halir, "Tilted subwavelength gratings: controlling anisotropy in metamaterial nanophotonic waveguides," Opt. Lett. 43, 4691-4694 (2018).

6. I. Staude and J. Schilling, "Metamaterial-inspired silicon nanophotonics," Nat. Photonics 11, 274-284 (2017).

7. S. Jahani, S. Kim, J. Atkinson, J. C. Wirth, F. Kalhor, A. Al Noman, W. D. Newman, P. Shekhar, K. Han, V. Van, R. G. DeCorby, L. Chrostowski, M. Qi, and Z. Jacob, "Controlling evanescent waves using silicon photonic all-dielectric metamaterials for dense integration," Nat. Commun. 9, 1893 (2018).

8. M. B. Mia, S. Z. Ahmed, I. Ahmed, Y. J. Lee, M. Qi, and S. Kim, "Exceptional coupling in photonic anisotropic metamaterials for extremely low waveguide crosstalk," Optica 7, 881-887 (2020).

9. H. Xu, D. Dai, and Y. Shi, "Anisotropic metamaterial-assisted allsilicon polarizer with 415-nm bandwidth," Photon. Res. 7, 1432-1439 (2019).

10. K. Chen, K. Yu, and S. He, "High performance polarization beam splitter based on cascaded directional couplers assisted by effectively anisotropic structures," IEEE Photon. J. 11, 1-9 (2019).

11. E. Hecht, Optics (Pearson Education/Addison-Wesley, 2002).

12. S. Jahani and $Z$. Jacob, "Transparent subdiffraction optics: nanoscale light confinement without metal," Optica 1, 96-100 (2014).

13. S. Jahani and Z. Jacob, "Photonic skin-depth engineering," J. Opt. Soc. Am. B 32, 1346-1353 (2015). 
14. S. Rytov, "Electromagnetic properties of a finely stratified medium," Sov. Phys. JETP 2, 466-475 (1956).

15. P. Cheben, R. Halir, J. H. Schmid, H. A. Atwater, and D. R. Smith, "Subwavelength integrated photonics," Nature 560, 565-572 (2018).

16. B. Smith, K. Suzuki, and J. Sheats, Microlithography: Science and Technology (Taylor \& Francis, 1998).

17. Lumerical Inc., https://www.lumerical.com/products/.

18. W.-P. Huang, "Coupled-mode theory for optical waveguides: an overview," J. Opt. Soc. Am. A 11, 963-983 (1994).

19. R. Halir, A. Maese-Novo, A. Ortega-Moñux, I. Molina-Fernández, J. Wangüemert-Pérez, P. Cheben, D.-X. Xu, J. Schmid, and S. Janz, "Colorless directional coupler with dispersion engineered sub-wavelength structure," Opt. Express 20, 13470-13477 (2012).

20. R. Halir, P. J. Bock, P. Cheben, A. Ortega-Moñux, C. Alonso-Ramos, J. H. Schmid, J. Lapointe, D.-X. Xu, J. G. Wangüemert-Pérez, Í. Molina-Fernández, and S. Janz, "Waveguide sub-wavelength structures: a review of principles and applications," Laser Photon. Rev. 9, 25-49 (2015).

21. W. J. Westerveld and H. P. Urbach, Silicon Photonics (IOP Publishing, 2017).

22. R. Halir, P. Cheben, J. M. Luque-González, J. D. SarmientoMerenguel, J. H. Schmid, G. Wangüemert-Pérez, D.-X. Xu, S. Wang, A. Ortega-Moñux, and Í. Molina-Fernández, "Ultra-broadband nanophotonic beamsplitter using an anisotropic sub-wavelength metamaterial," Laser Photon. Rev. 10, 1039-1046 (2016).
23. W. Bogaerts, D. Taillaert, B. Luyssaert, P. Dumon, J. Van Campenhout, P. Bienstman, D. Van Thourhout, R. Baets, V. Wiaux, and S. Beckx, "Basic structures for photonic integrated circuits in silicon-on-insulator," Opt. Express 12, 1583-1591 (2004).

24. Y. Xing, U. Khan, A. R. Alves Júnior, and W. Bogaerts, "Behavior model for directional coupler," in Proceedings Symposium IEEE Photonics Society Benelux (2017), pp. 128-131.

25. P. Virtanen, R. Gommers, T. E. Oliphant, M. Haberland, T. Reddy, D. Cournapeau, E. Burovski, P. Peterson, W. Weckesser, J. Bright, S. J. van der Walt, M. Brett, J. Wilson, K. Jarrod Millman, N. Mayorov, A. R. J. Nelson, E. Jones, R. Kern, E. Larson, C. Carey, I. Polat, Y. Feng, E. W. Moore, J. VanderPlas, D. Laxalde, J. Perktold, R. Cimrman, I. Henriksen, E. A. Quintero, C. R. Harris, A. M. Archibald, A. H. Ribeiro, F. Pedregosa, and P. van Mulbregt, and SciPy 1.0 Contributors, "SciPy 1.0: fundamental algorithms for scientific computing in Python," Nat. Methods 17, 261-272 (2020).

26. S. van der Walt, S. C. Colbert, and G. Varoquaux, "The NumPy array: a structure for efficient numerical computation," Comput. Sci. Eng. 13, 22-30 (2011).

27. W. McKinnon, D.-X. Xu, C. Storey, E. Post, A. Densmore, A. Delâge, P. Waldron, J. Schmid, and S. Janz, "Extracting coupling and loss coefficients from a ring resonator," Opt. Express 17, 18971-18982 (2009).

28. K. Han, S. Kim, J. Wirth, M. Teng, Y. Xuan, B. Niu, and M. Qi, "Stripslot direct mode coupler," Opt. Express 24, 6532-6541 (2016). 